\title{
Range-separation and the multiple radii functional approximation inspired by the strongly interacting limit of density functional theory
}

\author{
Tim Gould ${ }^{1}$ and Stefan Vuckovic ${ }^{2}$ \\ 1) Qld Micro- and Nanotechnology Centre, Griffith University, Nathan, Qld 4111, \\ Australia \\ ${ }^{2)}$ Department of Chemistry, University of California, Irvine, CA 92697, USA
}

\begin{abstract}
The strongly-interacting limit of density functional theory has attracted considerable attention recently due to its ability to deal with the difficult strong correlation problem. Recent work [JPCL 8, 2799-2805 (2017)] introduced the "multiple radii functional" (MRF) approximation, inspired by this limit, which is designed to work well for strong correlations between dissociated fragments. Here, we analyse the MRF in exactly solvable one-dimensional molecules, to uncover how it matches, and deviates from, exact results; and use range-separation of the Coulomb potential in both exact and approximate theory to explore how this varies in space. Our work opens a path to new approximations incorporating the MRF, amongst other ingredients.
\end{abstract}

PACS numbers: 31.15.ec,31.15.ep,03.65.Yz

\section{INTRODUCTION}

Density functional theory $(\mathrm{DFT})^{1}$ is presently a workhorse of electronic structure calculations across disciplines that range from biochemistry to material science. ${ }^{2-7}$ DFT is in principle an exact theory, but all practical calculations require an approximation to the exchange-correlation (xc) functional. This is a key quantity in DFT, as it captures the very quantum, Coulombic and fermionic nature of electrons. Density functional approximations (DFAs) to the xc functional have achieved a widespread success and nowadays they are very close to achieving the desired chemical accuracy for weakly and moderately correlated systems. ${ }^{7}$

In contrast, for strongly correlated systems the situation is strikingly different. A number of fundamental failures of DFAs when dealing with strong correlation have been described. ${ }^{2,8}$ The problem of strong correlation within DFAs has been looked at from several perspectives. ${ }^{2,3,8-10}$ Despite that, we still do not have a DFA able to correctly describe the physics of strong electronic correlations without compromising the accuracy of standard DFAs for weakly and moderately correlated systems. ${ }^{2,3,7,11}$

In attempt to address the long-standing strong correlation problem, Vuckovic and Gori-Giorgi recently proposed a new way for the construction of DFAs, ${ }^{12,13}$ inspired by the features of the exact $\mathrm{xc}$ functional from the strongly-interacting limit (SIL) of DFT. ${ }^{14-18}$ More specifically, they simplified and rescaled the xc functional from the SIL, and thus they made it suitable for the treatment of physical systems at a reasonable computational cost. In this way, they have laid the foundation for the so-called multiple radii functional (MRF). Already by construction, the MRF has several appealing properties missed by standard DFAs. It captures the physics of bond breaking, becomes highly accurate in the low-density limit, and provides accurate xc energy densities (defined within the conventional DFT gauge). ${ }^{11,19-21}$ These energy densities also display the correct asymptotic behaviour. ${ }^{12}$
While the development of efficient algorithms for an implementation of the MRF is currently subject to intensive efforts, its ultimate computational cost is expected to be comparable ${ }^{12}$ with that of local hybrid functionals, ${ }^{22-24}$ once efficient algorithms are designed. Initial tests of the MRF functional - on small atoms and the hydrogen molecule - show that the method captures strong correlation effects, while still being comparably accurate for weakly and moderately correlated electronic regimes. It thus is already a good general purpose density functional approximation (DFA).

So far, the the MRF functional has been applied to three-dimensional Coulombic systems. However, its framework can be easily transferred to other dimensionalities and other (isotropic) interactions between electrons. For this reason, as opposed to other DFAs (already starting from the local density approximation ${ }^{1}$ ), the MRF does not require re-parametrization when a number of dimensions and/or an interaction between particles are changed. This is because the functional is based on the effect of local descriptors of electron numbers (see next section) on interactions combined with explicit interaction terms, and is consequently insensitive to short-range expansion of homogeneous electron gases.

In the present work, we draw on this feature of MRF and apply it to an exactly solvable one-dimensonal (1D) molecular models with two electrons interacting via softCoulomb potential. Such 1D models have served as a convenient tool for testing new DFAs ${ }^{25-27}$ and used as a theoretical laboratory for revealing features of the exact xc functional. ${ }^{28-30}$ Notably, they offer the chance to tune inter-species interactions from strong to weak in a controlled fashion with numerically exact solutions.

We perform an analysis at the pair-density level where we translate the exact pair-densities into their MRF form and compare them with those obtained from the MRF approximation. We also use here the common decomposition of the Coulomb (in our case soft-Coulomb) electronic interaction into a short-range (sr) and long-range (lr) part, ${ }^{31-34}$ to study the underlying sr and lr compo- 


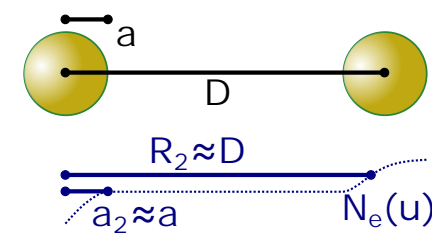

FIG. 1. Illustration of MRF in $\mathrm{H}_{2}$.

nents of the MRF xc energy densities. This is an important first step in combining MRF with popular rangeseparation techniques. ${ }^{31}$

The paper is organized as follows. First, we introduce the theory behind the MRF. Then we introduce the 1D model systems we use to explore it. Then, we study exact and approximate pair-densities. Next, we discuss results by studying exact and approximate energy densities, in the context of range-separation. Finally, we conclude.

\section{THEORY}

\section{A. Basic DFT background}

We begin by briefly reviewing basic DFT equations that concern the density-fixed adiabatic connection (AC) representation of the xc functional. ${ }^{35,36}$ Consider the DFT wavefunction obtained from a constrained minimisation: ${ }^{37}$

$$
\Psi_{\lambda}[n]=\underset{\Psi \rightarrow n}{\arg \min }\langle\Psi|\hat{T}+\lambda \hat{U}| \Psi\rangle .
$$

The corresponding pair-density is given by:

$$
\begin{aligned}
& P_{2}^{\lambda}\left(\mathbf{r}, \mathbf{r}^{\prime}\right)=N(N-1) \times \\
& \sum_{\sigma_{1} \ldots \sigma_{N}} \int\left|\Psi_{\lambda}\left(\mathbf{r} \sigma_{1}, \ldots, \mathbf{r}_{N} \sigma_{N}\right)\right|^{2} \mathrm{~d} \mathbf{r}_{3} \ldots \mathrm{d} \mathbf{r}_{N}
\end{aligned}
$$

and the underlying xc hole is given by:

$$
h_{\mathrm{xc}}^{\lambda}\left(\mathbf{r}, \mathbf{r}^{\prime}\right)=\frac{P_{2}^{\lambda}\left(\mathbf{r}, \mathbf{r}^{\prime}\right)}{n(\mathbf{r})}-n\left(\mathbf{r}^{\prime}\right),
$$

The electrostatic potential of the xc hole provides a definition (i.e., with the conventional DFT gauge ${ }^{11,38,39}$ ) for the xc energy density: of the xc hole:

$$
w_{\lambda}(\mathbf{r})=\frac{1}{2} \int h_{\mathrm{xc}}^{\lambda}\left(\mathbf{r}, \mathbf{r}^{\prime}\right) U\left(\left|\mathbf{r}-\mathbf{r}^{\prime}\right|\right) \mathrm{d} \mathbf{r}^{\prime}
$$

Then we can write the xc functional in terms of $w_{\lambda}(\mathbf{r})$ as:

$$
E_{\mathrm{xc}}[n]=\int_{0}^{1} d \lambda \int d \mathbf{r} n(\mathbf{r}) w_{\lambda}(\mathbf{r})
$$

\section{B. The MRF functional}

Next, we summarise the key features of the MRF. In contrast to traditional density functional approximations, which use (semi)local quantities and Kohn-Sham (KS) orbitals, ${ }^{4,40,41} \mathrm{MRF}$ approximations involve integrated quantities of the density. Specifically, they build from the number of electrons,

$$
N_{e}(u ; \mathbf{r})=\int \Theta\left(\left|\mathbf{r}-\boldsymbol{r}^{\prime}\right|-u\right) n\left(\boldsymbol{r}^{\prime}\right) d \boldsymbol{r}^{\prime}
$$

within a given radius $u$ around a point $\mathbf{r}$.

This quantity is naturally suited to strong correlation problems. Its advantage can be understood by considering two well-separated $\mathrm{H}$ atoms (dissociated $\mathrm{H}_{2}$ ), which must not interact with a Coulomb potential since both atoms are neutral. By taking $a<u<D$ (where $a$ is the radius of an $\mathrm{H}$ atom and $D$ is the distance between nucleii) we see that $N_{e}(u ; \mathbf{r}) \approx 1$ for $\mathbf{r}$ near each nucleus. This function can thus accommodate the $-1 / D$ net attraction between electrons and electrons and nucleii, to balance out the $1 / D$ repulsion between the two nucleii. Approximations to the correlation energy can thereby be built from $N_{e}$ to give DFAs which correctly accommodate the long-range interactions.

Vuckovic and Gori-Giorgi ${ }^{12}$ recently introduced an approximation that uses $N_{e}(u ; \mathbf{r})$ to capture the behaviour described above. Their approximation involves calculating

$$
\begin{aligned}
a_{i}(\mathbf{r}) & =N_{e}^{-1}(i-1 ; \mathbf{r}) \\
S_{i}(\mathbf{r}) & =\left.\frac{\partial N_{e}(u ; \mathbf{r})}{\partial u}\right|_{u=a_{i}(\mathbf{r})} \\
\sigma_{i}(\mathbf{r}) & \approx \frac{1}{2} e^{-b S_{i}(\mathbf{r})^{2}} \\
R_{i}(\mathbf{r}) & =N_{e}^{-1}\left(i-1+\sigma_{i} ; \mathbf{r}\right),
\end{aligned}
$$

which are, respectively: the radius $a_{i}$ of a sphere around point $\mathbf{r}$ which contains exactly $i-1$ electrons; the rate $S_{i}$ at which that radius is changing; a small fluctuation $\sigma_{i}$ that reflects how slowly the radius changes, and which can thus identify regions which are free of electrons; the radius containing $i-1+\sigma_{i}$ electrons.

Finally, the xc functional within the MRF approximation is written as

$$
\begin{aligned}
E_{\mathrm{xc}}^{\mathrm{MRF}} & =T_{c}[n]+\int d \mathbf{r} n(\mathbf{r}) w_{1}^{\mathrm{MRF}}(\mathbf{r}), \\
w_{1}^{\mathrm{MRF}}(\mathbf{r}) & =\frac{1}{2} \sum_{i=2}^{N} U\left(R_{i}(\mathbf{r})\right)-\frac{1}{2} v_{\mathrm{H}}(\mathbf{r}),
\end{aligned}
$$

where $U(R)$ is the Coulomb interaction between electrons at distance $R$ i.e. $1 /\left|\mathbf{r}-\boldsymbol{r}^{\prime}\right|$ typically, and where $v_{\mathrm{H}}(\mathbf{r})$ is the Hartree potential. We remark that $\int d \mathbf{r} n(\mathbf{r}) w_{1}^{\mathrm{MRF}}(\mathbf{r})$ of eq. 12 does not contain $T_{c}[n]$, the kinetic energy contribution to the xc energy. This is also the case with the exact xc functional from the SIL, to which the MRF 
is inspired. However, one can in principle define the $\lambda$ dependent $\sigma_{i}^{\lambda}(\mathbf{r})$ and by integration of the corresponding $w_{\text {MRF }}^{\lambda}[n]$ from 0 to 1 , one can recover $T_{c}$. Such a concept was recently discussed by Vuckovic ${ }^{13}$. Nevertheless, for simplicity we restrict here to the analysis of the $\lambda=1$ case.

Let us briefly return to dissociated $\mathrm{H}_{2}$ to consider how the approximation correctly captures asymptotic behaviours. We have two electrons, and thus only $i=2$ contributes to the MRF. As before we use $a$ as the radius of an $\mathrm{H}$ atom, and $D \gg a$ as the distance between them. We thus find, for $\mathbf{r}$ in the vicinity of atom $\mathrm{H}_{A}$

$$
\begin{array}{ll}
a_{2}\left(\mathbf{r} \in \mathrm{H}_{A}\right) \approx a, & S_{2}\left(\mathbf{r} \in \mathrm{H}_{A}\right) \approx 0 \\
\sigma_{2}\left(\mathbf{r} \in \mathrm{H}_{A}\right) \approx \frac{1}{2}, & R_{2}\left(\mathbf{r} \in \mathrm{H}_{A}\right) \approx D .
\end{array}
$$

Some of these quantities are illustrated in Figure 1

The finding that $R_{2} \approx D$ follows from the fact that we can only capture an additional half an electron by extending our sphere well into the region containing $\mathrm{H}_{B}$. Finally, for the sum of the Hartree and xc energy, which we denote here by $E_{\mathrm{Hxc}}$, we get:

$$
E_{\mathrm{Hxc}}^{\mathrm{MRF}}=2 \int_{\mathrm{H}_{A}} d \mathbf{r} n(\mathbf{r}) \frac{1}{2} \frac{1}{D}=\frac{1}{D}
$$

where we used $T_{c}=0$ for the $\mathrm{H}$ atom. The pre-factor 2 comes from the fact that we exploited the symmetry of the problem to integrate only in the region of $\mathrm{H}_{A}$. Adding this result to $1 / D$ from the nucleii, and $-2 / D$ from the electron-nuclear interactions gives a net zero long-range interaction, as desired.

\section{RESULTS}

We now turn to results. Before doing so, we describe the computational procedure employed and give the precise description of the one-dimensional model that we use.

Before proceeding, however, we will briefly reiterate why the one-dimensional model is a useful test bed for theories, despite the fact that it can only mimic real chemistry and it does not have the richness of threedimensional structures. Despite these limitations, onedimensional models have the following points in their favour: i) they can provide a tunable connection between strongly and weakly correlated systems, in a fashion that is clearly representative of three-dimensional equivalents $^{25,27,28,42}$; ii) they can be efficiently solved exactly (to within a desired numerical accuracy) for both the interacting and non-interacting (KS) systems; iii) they allow for direct visulaistion due to the reduced dimension. They are thus more than sufficient to illustrate key features of the approximations we make here.

\section{A. Computational details}

The Hamiltonian of our 1D molecular model with two electrons is:

$$
\hat{H}=\hat{t}_{1}+\hat{t}_{2}++v\left(x_{1}\right)+v\left(x_{2}\right)+U\left(\left|x_{1}-x_{2}\right|\right),
$$

where our electrons interact via soft-Coulomb potential:

$$
U(u)=\frac{1}{\sqrt{1+u^{2}}} .
$$

Here, $\hat{t}_{1 / 2}=\frac{-1}{2} \frac{\partial^{2}}{\partial x_{1 / 2}^{2}}$, and we employ the following external potential:

$$
\begin{aligned}
v(x)= & -U(x+D / 2) \\
& -\left[U(x-D / 2)+\eta e^{-(x-D / 2)^{2}}\right],
\end{aligned}
$$

where $D$ is the bond length of our molecule, which consists of a left atom placed at $-D / 2$ and right atom at $+D / 2$.

The role of $\eta$ is to control the well depth on the right atom - the well becomes deeper as $\eta$ increases, giving an effective local charge of $Z=1+\eta$ for the "nucleus", which correctly reduces to $Z=1$ at longer distances. The case $Z=1(\eta=0)$ is representative of the strongly-correlated $\mathrm{H}_{2}$ diatom dissociation process, whereas $Z \geq 1.5\left(\eta \geq \frac{1}{2}\right)$ is more similar to dissociation of an ionic diatom like $\mathrm{LiH}$.

Finding the (real) eigenstates $\Psi^{(k)}\left(x, x^{\prime}\right)$ (and energies $\left.E^{(k)}\right)$ of the two-electron Hamiltonian of Eq 16 is equivalent to finding the eigenstate of a Hamiltonian with one electron moving in a two-dimensional potential. Thus, we employ simple numerics implemented in Python to find groundstate wavefunctions $\Psi^{(0)}\left(x, x^{\prime}\right)$ of Eq. 16, from which we can obtain the density $n(x)=2 \int \Psi^{(0)}\left(x, x^{\prime}\right)^{2} d x^{\prime}$ and pair-density $P_{2}\left(x, x^{\prime}\right)=$ $2 \Psi^{(0)}\left(x, x^{\prime}\right)^{2}$.

\section{B. Setting the stage}

Once we have obtained the density $n(x)$ and the physical pair-density $P_{2}^{\lambda=1}\left(x, x^{\prime}\right)$ we can obtain a wide range of energy properties. This includes the xc energy densities at $\lambda=1$ which will be compared with those from the MRF model. Firstly, for $N=2$ unpolarised systems it is trivial to obtain the exchange energy density from:

$$
w_{\mathrm{x}}(x) \equiv w_{\lambda=0}(x)=-\frac{1}{4} v_{\mathrm{H}}(x) .
$$

From these we can obtain:

$$
\begin{aligned}
w_{\mathrm{c}, 1}(x) & =w_{1}(x)-w_{0}(x), \\
w_{\mathrm{c}, \mathrm{MRF}}(x) & =w_{1}^{\operatorname{MRF}}(x)-w_{0}(x),
\end{aligned}
$$

for the correlation-only energy densities. 

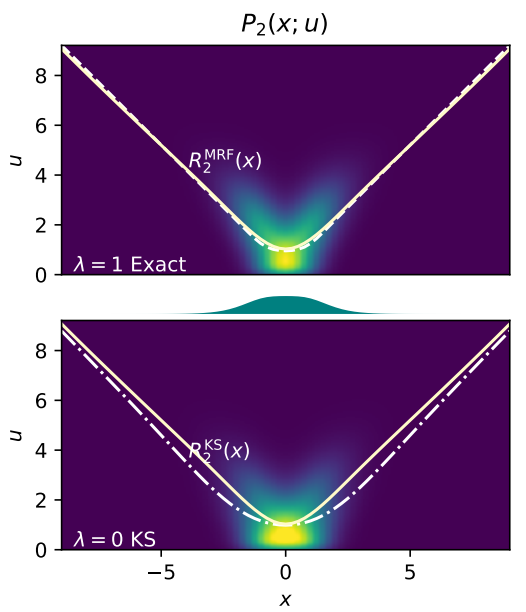
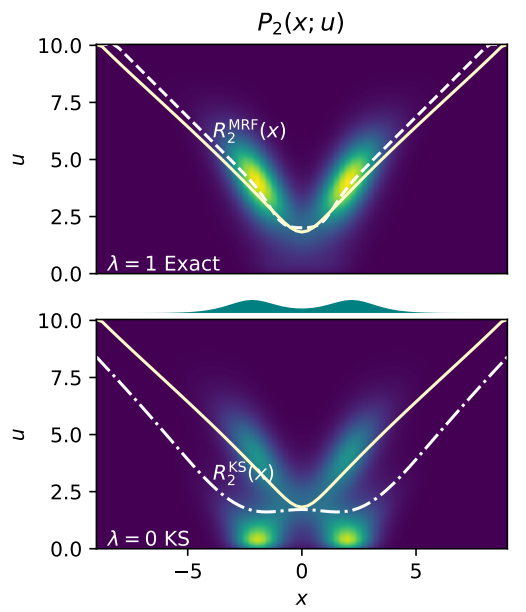
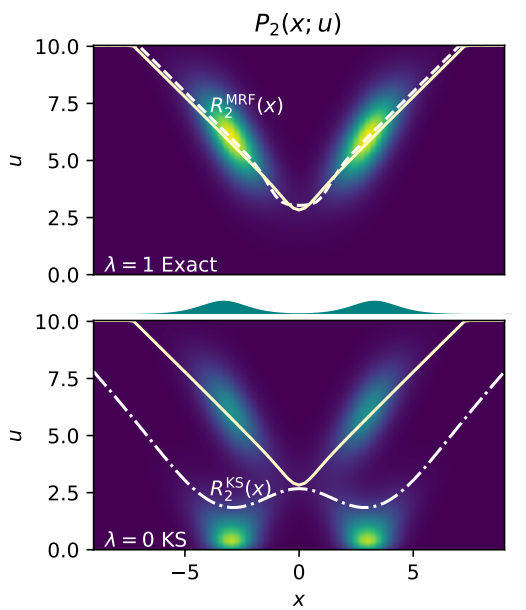

FIG. 2. Plots of $P_{2}(x ; u)$ for $D=2,4,6$ (left to right) with $Z=1(\eta=0.0)$. We show the interacting (top) and Kohn-Sham (bottom) pair-densities, with navy indicating no (pair)-density and brighter colours indicating increased contributions. Plots include $R_{2}^{\lambda=1}(x)$ obtained from the exact calculation (solid cream line); $R_{2}^{\mathrm{MRF}}(x)$ to represent $P_{2}^{\mathrm{MRF}}(x ; u)$ (white dashes, top only); and $R_{2}^{\mathrm{KS}}=R_{2}^{\lambda=0}$ to represent a traditional KS picture (white dot-dash, bottom only). The density is shown in teal between the plots, to highlight the regions which contribute to the total energy.
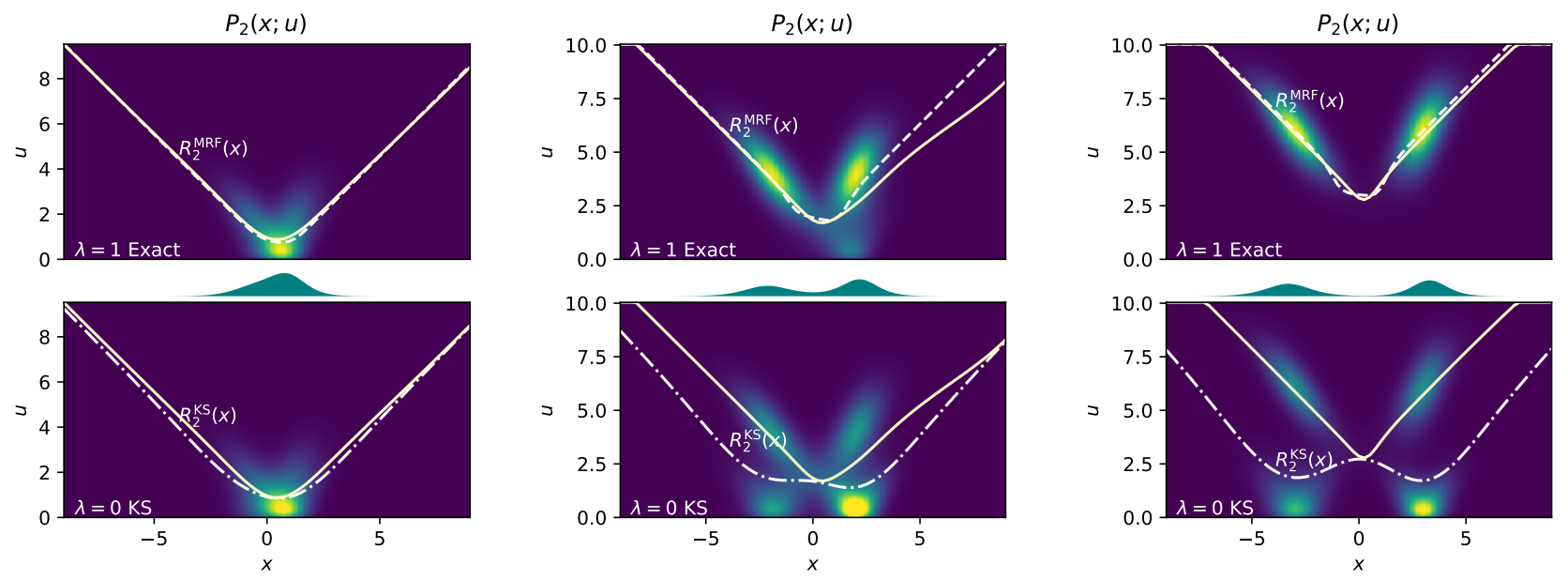

FIG. 3. Like Fig. 2 but for $Z=1.5(\eta=0.5)$.

In addition to having easy-to-obtain exact solutions, both for interacting and non-interacting systems, twoelectron systems offer another advantage - the MRF system can be reverse engineered, so that we can compare the results of approximations against their values obtained from exact theory.

For $N=2$, we can use exact $w_{\lambda=1}(x)$ (obtained from the ground state of Eq. 16) to obtain the exact $R_{i}^{\lambda=1}(x)$. We do this by inverting Eq. 12 to obtain:

$$
R_{2}^{\lambda=1}(x)=U^{-1}\left(v_{H}(x)+2 w_{\lambda=1}(x)\right)
$$

From the radius of Eq. 22, we can obtain the exact $\sigma_{i}^{\lambda}(\mathbf{r})$, which will be given by:

$$
\sigma_{2}^{\lambda=1}(x)=N_{e}\left(R_{2}^{\lambda=1}(x) ; x\right)-1 .
$$

With all key quantities defined, including exact ones, we are now ready to dig deeper into the MRF to study how well it works, and how it might be improved.

\section{MRF and pair-densities}

To begin, we explore the pair-density. For a given $\Psi$, the electronic repulsion energy $V_{e e}$ is explicitly given in terms of its pair-density. Thus, for $V_{e e}^{\lambda}[n]=$ $\left\langle\Psi_{\lambda}[n]|\hat{U}| \Psi_{\lambda}[n]\right\rangle$, we have:

$$
V_{e e}^{\lambda}[n]=\int \frac{d \mathbf{r} d \mathbf{r}^{\prime}}{2} U\left(\mathbf{r}, \mathbf{r}^{\prime}\right) P_{2}^{\lambda}\left(\mathbf{r}, \mathbf{r}^{\prime}\right) .
$$


Then, invoking the adiabatic connection (as in eq. 5), we can write Hxc energy as: $E_{\mathrm{Hxc}}[n]=\int_{0}^{1} d \lambda V_{e e}^{\lambda}[n]$. However, we focus here on the $\operatorname{KS}(\lambda=0)$ and fullyinteracting cases $(\lambda=1)$, rather than the $\lambda$-averaged quantities.

Furthermore, since the electronic interaction depends only on the distance between particles, we can rewrite (24) as:

$$
V_{e e}^{\lambda}[n]=\int \frac{d \mathbf{r} d u}{2} U(u) P_{2}^{\lambda}(\mathbf{r} ; u),
$$

where $P_{2}^{\lambda}(\mathbf{r} ; u)$ is the "radially" averaged pair-density

$$
P_{2}^{\lambda}(\mathbf{r} ; u)=\int d \mathbf{r}^{\prime} P_{2}^{\lambda}\left(\mathbf{r}, \mathbf{r}^{\prime}\right) \delta\left(u-\left|\mathbf{r}-\mathbf{r}^{\prime}\right|\right),
$$

at point $\mathbf{r}$ and radius $u$. The equivalent $V_{e e}^{\lambda=1}$ energy expression for the MRF [using (12)] is:

$$
\begin{aligned}
V_{e e}^{\mathrm{MRF}} & =\int \frac{d \mathbf{r}}{2} n(\mathbf{r}) \sum_{i \geq 2} U\left(R_{i}^{\mathrm{MRF}}(\mathbf{r})\right) \\
& \equiv \int \frac{d \mathbf{r} d u}{2} U(u) n(\mathbf{r}) \sum_{i \geq 2} \delta\left(u-R_{i}^{\mathrm{MRF}}(\mathbf{r})\right),
\end{aligned}
$$

where $\delta$ is the Dirac delta function. Thus, the effective pair-density in MRF is

$$
P_{2}^{\mathrm{MRF}}(\mathbf{r} ; u)=n(\mathbf{r}) \sum_{i \geq 2} \delta\left(u-R_{i}^{\mathrm{MRF}}(\mathbf{r})\right) .
$$

In one-dimension $P_{2}^{\lambda}(\mathbf{r} ; u)$ becomes $P_{2}^{\lambda}(x ; u)$ and $P_{2}^{\mathrm{MRF}}(\mathbf{r} ; u)$ becomes $P_{2}^{\mathrm{MRF}}(x ; u)=n(x) \sum_{i \geq 2} \delta(u-$ $\left.R_{i}^{\mathrm{MRF}}(x)\right)$.

The MRF pair-density of of Eq. 28 is inspired by the form of the exact SIL pair-densities. Since it is given by the sum of $\delta$ functions one can easily object that this pairdensity cannot represent its physical counterpart. However, as discussed in Ref. $13, P_{2}^{\mathrm{MRF}}(\mathbf{r} ; u)\left[\right.$ or $\left.P_{2}^{\mathrm{MRF}}(x ; u)\right]$ serves in the MRF framework as the auxiliary object with the purpose of delivering accurate xc energy densities (to be considered later). In this context, eq. (28) can be made to yield exact xc energy densities by replacing the radii $R_{i}$ by their exact counterparts, obtainable for two electron systems via eq. (22).

In Figures 2 and 3 we show exact $P_{2}^{\lambda=1}$ (top panels) and KS $P_{2}^{\lambda=0}$ (bottom panels) as a function of $x$ and $u$ for a selection of 1D molecules. We include on these figures $R_{2}(x)$, obtained from Eq. (10), (white dashed lines, top panels only), which for our two electron systems is the only contributing radius in the MRF. For comparison, lines representing the exact $R_{2}^{\lambda=1}(x)$ (solid cream lines) and $R_{2}^{\mathrm{KS}}=R_{2}^{\lambda=0}$ (white dot-dash, bottom panels only), obtained from Eq. 22, are also shown. As the effective MRF pair-potential $P_{2}^{\mathrm{MRF}}(x ; u)$ is scaled by $n(x)$, we also show the density (between the panels - teal) for reference.

We see immediately that the MRF radius passes through the centre of the high-charge-density regions of
$P_{2}(x ; u)$ in almost all cases, which is consistent with its exact counterpart. This means it is able to reproduce, on average, the interactions between regions, which becomes more accurate as the regions are further apart in the dissociation limit.

In contrast, the KS pair-density has weak contributions at this point, and a more significant contribution for $u \rightarrow 0$. This second, unphysical contribution comes from the failure of a single-reference system to reproduce the multi-reference physics of strong correlations. ${ }^{2,8}$ It manifests in our figures as a very poor effective radius $R_{2}^{\mathrm{KS}}$, as shown in dot-dashes in the lower panels.

The one case where MRF struggles a little is with $\eta=0.5$ and $D=4$ (top middle panel of Figure 3), where the bond breaking leads to an asymmetry in charge between the left and right atoms. In this case The MRF radius (the dashed line) is not as asymmetric as its exact counterpart (cream solid line). This is a particularly challenging case, however, as in the dissociation limit there is only a small energy gap between the strongly-correlated ground-state and the charge transfer excited state. In the interim region $(D=4)$ shown here, these two states are in superposition and are thus extremely difficult to model.

\section{Exchange/correlation energy densities}

Let us now understand how the MRF works. Returning to the exact and approximate solutions, we recognise that $\sigma_{2}^{\lambda}(x)$ [eq. (9)] is a more physically relevant quantity than the radius $R_{2}^{\lambda}(x)$. The $\sigma_{2}$ quantity physically represents the deviation from the expected charge associated with the effective distances between electrons, to be explained in more detail below. The key idea is that for $N=2, R_{2}^{\lambda=1}(x)$ represents the effective distance between electrons (i.e. the distance required to ensure a Coulomb potential giving the correct potential $v_{H}+2 w_{\lambda=1}$; and $\nu_{2}^{\lambda=1}=N_{e}\left(R_{2}^{\lambda=1}(x) ; x\right)$ represents the effective charge at that distance. Thus, $\sigma_{2}=\nu_{2}-1$ [see (10) and (23)] is the deviation from the expected effective charge $\left(\nu_{i}^{\lambda=1}=i-1\right)$.

In Figures 4 and 5, we show plots of the exact $\sigma_{2}^{\lambda}(x)$ at $\lambda=0$ and $\lambda=1$ obtained from Eqs. (22) and (23) for 1D densities from the ground state of Eq 16 for different $D$ and $\eta$ values [Eq. (18)]. These exact $\sigma^{\lambda}(x)$ curves are compared with those obtained from the approximation to $\sigma^{\lambda}(x)$ of Eq. (10). Insets of the same figure compare the exact $w_{\mathrm{c}, \lambda}(x)$ at $\lambda=1$ with $w_{\mathrm{c}, 1}^{\mathrm{MRF}}$ of MRF.

As can be seen from Figures 4 and 5 , the approximate $\sigma^{\lambda=1}(x)$ of Eq. 10 is in all cases closer to the exact $\sigma^{\lambda}(x)$ at $\lambda=1$ than at $\lambda=0$. This confirms, at least qualitatively, that the ansatz of Eqs. 7 and 10 is well-suited for the description of the electronic interactions at the physical regime. We can also observe that $\sigma^{\lambda=1}(x)$ of Eq. 10 becomes more accurate as the bond length increases, indicating that it is working to describe strong correlations. 

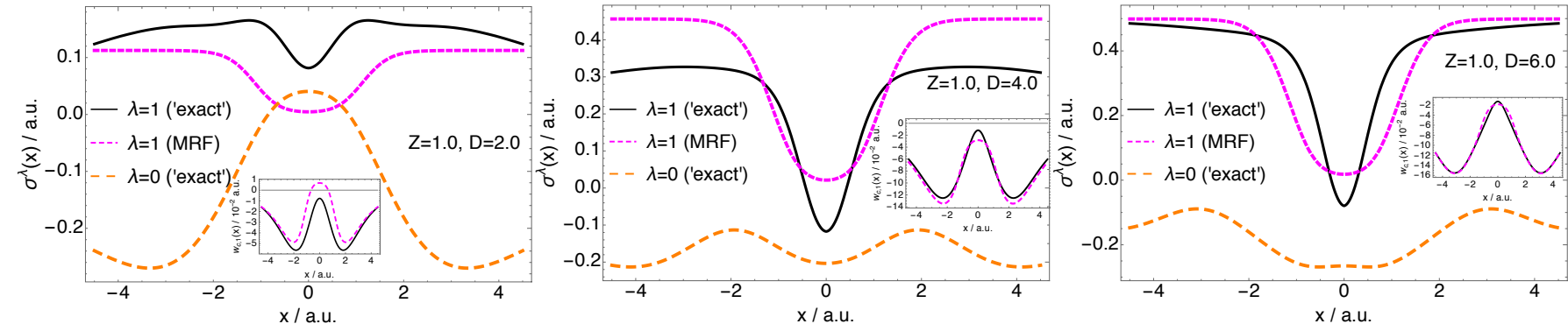

FIG. 4. Plots showing exact $\sigma_{2}^{\lambda}(x)$ quantity for different values of $D$ at $Z=1$ [the ground state $n(x)$ of Eq (16)] with $\lambda=0$ and $\lambda=1$ [obtained from Eqs. (22) and (23)]. Approximate MRF $\sigma_{2}^{\lambda}(x)$ at $\lambda=1$ has been obtained from Eq. (10) with $b=5$. Insets compare the exact and MRF correlation energy density at $\lambda=1$ [eqs. (20),(21)].
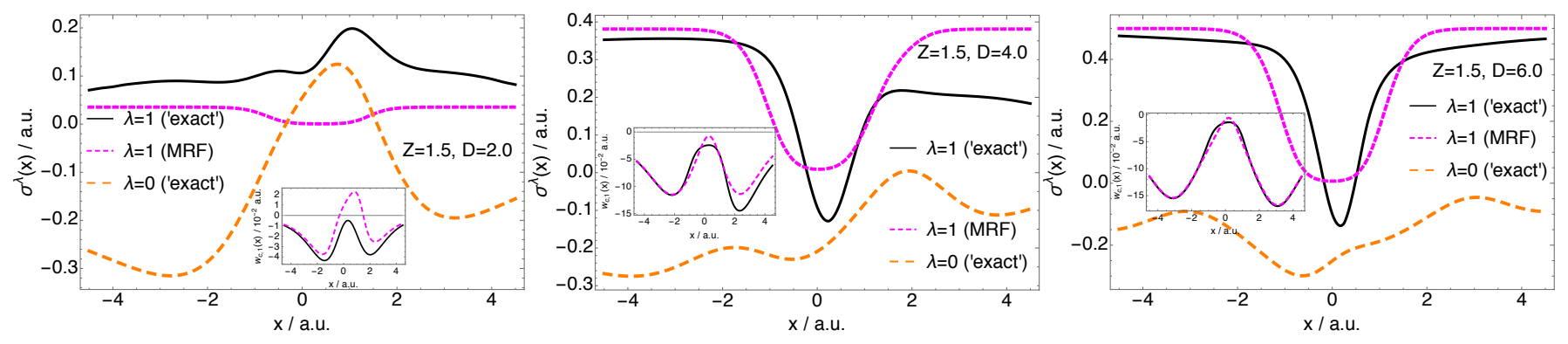

FIG. 5. The same plots as in Figure 4, but at $Z=1.5$, or $\eta=0.5$.

One unappealing feature of the approximate $\sigma^{\lambda=1}(x)$ arising from Eq. 10 is that it lies below $\sigma_{2}^{0}(x)$ in the bond midpoint region (in both Figures 4 and 5 when $D=2$ ). For this reason, the correlation component $w_{\mathrm{c}, 1}^{\mathrm{MRF}}(\mathbf{r})$ becomes unphysically positive in this region (see the insets of Figures 4 and 5). This feature has also been observed in inter-shell regions of real atoms ${ }^{13}$.

Next, we analyse the origin of these features in more detail, by range-separating the xc energy contributions into short-range (sr) and long-range (lr) components.

\section{E. Range-separation of MRF}

To gain more insight into the accuracy of the MRF functional, we compare its range-separation resolved xc energy densities with its exact counterparts. Here, we carry out the range-separation only at the energy level. That is, we apply it to key quantities like pair-densities $P_{2}^{\lambda=1}\left(\mathbf{r}, \boldsymbol{r}^{\prime}\right)$ and the corresponding xc hole, $h_{\mathrm{xc}}^{\lambda=1}\left(\mathbf{r}, \boldsymbol{r}^{\prime}\right)$.

Range-separation involves dividing key terms (usually energies) into short-range (sr) and long-ranged (lr) components, for the purpose of understanding and/or approximating them separately. Here, we use it to partition the total energy density, $w_{\lambda}(\mathbf{r})=w_{\lambda}^{\mathrm{sr}}(\mathbf{r})+w_{\lambda}^{\mathrm{lr}}(\mathbf{r})$, into short- and long-range components.

To define the sr and lr components for our 1D systems, we first partition the soft-Coulomb potential:

$$
U(u)=U^{\mathrm{sr}}(u)+U^{\mathrm{lr}}(u)
$$

using $U^{\mathrm{sr}}(u)=U(u) e^{-\mu^{2} u^{2}}$, where $\mu$ is a parameter with inverse length dimension. This lets us decompose $w_{\lambda}(\mathbf{r})$ [using eq. (3)] into sr and lr contributions:

$$
\begin{aligned}
w_{\lambda}(\mathbf{r}) & =\underbrace{\frac{1}{2} \int h_{\mathrm{xc}}^{\lambda}\left(\mathbf{r}, \mathbf{r}^{\prime}\right) U^{\mathrm{sr}}\left(\left|\mathbf{r}-\mathbf{r}^{\prime}\right|\right) \mathrm{d} \mathbf{r}^{\prime}}_{w_{\lambda}^{\text {sr }}(\mathbf{r})} \\
& +\underbrace{\frac{1}{2} \int h_{\mathrm{xc}}^{\lambda}\left(\mathbf{r}, \mathbf{r}^{\prime}\right) U^{\mathrm{Ir}}\left(\left|\mathbf{r}-\mathbf{r}^{\prime}\right|\right) \mathrm{d} \mathbf{r}^{\prime}}_{w_{\lambda}^{\text {sr }}(\mathbf{r})}
\end{aligned}
$$

This separation can also be applied to the $\lambda$-dependent correlation energy density $w_{\mathrm{c}, \lambda}(\mathbf{r})=w_{\lambda}(\mathbf{r})-w_{0}(\mathbf{r})$ (in terms of the electrostatic potential of the correlation hole), to obtain its range-separated contributions $w_{\mathrm{c}, \lambda}^{\mathrm{sr}}(\mathbf{r})=w_{\lambda}^{\mathrm{sr}}(\mathbf{r})-w_{0}^{\mathrm{sr}}(\mathbf{r})$ and $w_{\mathrm{c}, \lambda}^{\mathrm{lr}}(\mathbf{r})=w_{\lambda}^{\mathrm{lr}}(\mathbf{r})-w_{0}^{\mathrm{lr}}(\mathbf{r})$.

Before reporting any tests, let us first take a moment to consider and clarify the effective range of strong correlation. Strong correlation that pertains to bond dissociation of systems that dissociate into fragments with degenerate KS states, such as $\mathrm{H}_{2}$ or $\mathrm{LiH}$, is typically considered to be a long-range phenomenon. From the perspective of the pair-density this is indeed the case, as can be seen clearly in Figures 2 and 3 by considering that the highest density (in yellow) occurs for $u \approx D$, i.e. on the other atom. Thus, one might argue that strong correlations are a long-range phenomenon.

However, from the perspective of the $\mathrm{xc}$ hole and its electrostatic potential (i.e. the xc energy density), in energetically important regions this phenomenon is 

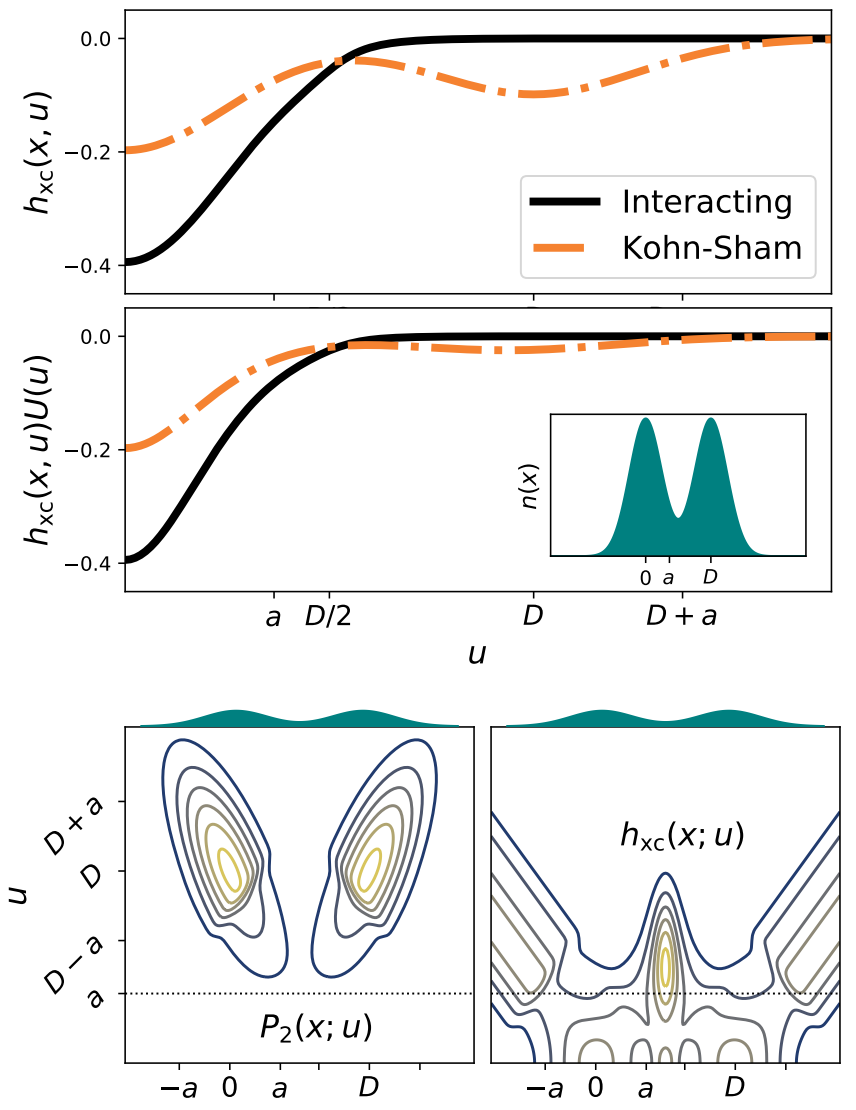

FIG. 6. Illustration of the short-rangedness of the hole density. Top panels show $h_{\mathrm{xc}}^{\lambda}(x, u)$ and $h_{\mathrm{xc}}^{\lambda}(x, u) U(u)$ at $\lambda=0$ ('Kohn-Sham' $-h_{\mathrm{x}}$ ) and $\lambda=1$ ('interacting'). Although strong correlation is considered to be a long-range phenomena, the effect on the xc hole and energy densities are mostly short- (or mid-) ranged where it matters for energies. Key length scales (per Figure 1) are indicated, and $x$ is chosen to lie on one of the nucleii. The density is shown in teal in an inset. The bottom panels show contours of $P_{2}(x ; u)$ and $h_{\mathrm{xc}}(x ; u)$, to further highlight the short-rangedness of the latter quantity. The origin is chosen, as in the top panel, to lie on the left nucleus - the other nucleus is at $D$.

mostly short- and mid-ranged. Take for an example the stretched real (i.e. 3D) hydrogen molecule. If we place a reference electron in vicinity of one of the two nuclei the xc hole localizes around this nucleus so it removes an electron that surrounds it. ${ }^{43,44}$. This feature is why (e.g.) generalized gradient approximations (GGAs) suffer less than Hartree-Fock theory from dissociation errors: the semi-local hole in a GGA is always short-ranged, unlike the exchange hole which can delocalize across multiple species. ${ }^{45}$

To illustrate this point more directly, let us return to our 1D model system for partially dissociated $\mathrm{H}_{2}$. Figure 6 compares the "radially" averaged xc hole:

$$
h_{\mathrm{xc}}^{\lambda}(x ; u)=\int d x^{\prime} h_{\mathrm{xc}}^{\lambda}\left(x, x^{\prime}\right) \delta\left(u-\left|x-x^{\prime}\right|\right),
$$

at $\lambda=1$ against its $\mathrm{KS}$ counterpart $\left(\lambda=0: h_{\mathrm{x}}\right)$, using Eq. (3). Note that the key length scales, such as $a$ and $D$, are also indicated in this figure, allowing us to clearly see that the effect on the xc hole and energy densities are short- (or mid-) ranged. The bottom panels further illustrate the point by showing the pair-density $P_{2}$ (clearly long-ranged) and hole density $h_{\mathrm{xc}}$ (short-ranged where it counts, i.e. where the density is significant).

Now we are ready to analyse the MRF from a rangeseparated perspective. In Figure 7 we thus compare sr $\left[w_{\lambda}^{\mathrm{sr}}(\mathbf{r})\right]$ and $\operatorname{lr}\left[w_{\lambda}^{\mathrm{lr}}(\mathbf{r})\right]$ energy densities for both MRF and exact theory. These energy densities have been evaluated on $n(x)$ pertaining to $v(x)$ of $\mathrm{Eq} 18$, with $Z=1$ and $D=2$ a.u., $Z=1, D=6$, and $Z=1.5, D=6$. We set $\mu=0.6$, corresponding to damping over a length scale 1.7 Bohr radii, a similar scale to that used on common functionals like HSE. ${ }^{33}$

First, we focus the lr and sr xc energy densities at $D=2$ a.u. Interestingly, even in this case, where the overall accuracy of $w_{1}^{\mathrm{MRF}}(x)$ is ow compared to larger $D$, its sr component is still in a good agreement with the corresponding exact energy density.

We can also see from Figure 7 that the long-range MRF energy density for the two strongly correlated model systems corresponding to $D=6$ (at both $Z=1.0$ and $Z=1.5$ ), the sr MRF xc energy density is more accurate than its lr part, most obviously seen in the light purple shaded curves. This is despite the fact that the lr part is much smaller in absolute energy than the sr part, meaning the error in the sr component is smaller in absolute terms, and significantly smaller in relative terms. Given that the MRF functional has been designed to capture the physics of bond dissociation, this makes sense in our picture based on the short-rangedness of the xc hole.

We next observe that in all cases in Figure 7, the $\mathrm{lr}$ MRF xc energy densities (the less accurate of the two range-separation resolved components) again become accurate at large $|x|$, due to the fact that MRF xc energy densities display the correct asymptotic behavior. ${ }^{13}$. We remark that by virtue of our $U^{\text {sr }}$ and $U^{\text {lr }}$ definitions, $w_{1}^{\mathrm{lr}}(x)$ behaves asymptotically as $w_{1}(x):-\frac{1}{2|x|}(|x| \rightarrow \infty)$, whereas $w_{1}^{\mathrm{sr}}(x)$ decays much faster.

Staying with Figure 7, we can also see that for $Z=1$ and $D=6$ a.u., both sr and lr components of $w_{1}^{\mathrm{MRF}}(x)$ are in closer agreement with the exact energy densities. Even in this stretched case, we can see that the sr component of $w_{1}^{\mathrm{MRF}}(x)$ is again more accurate than the corresponding $\mathrm{lr}$ component. The same result holds for $Z=1.5$ and $D=6$ a.u., highlighting the accuracy of MRF in the dissociation limit.

\section{F. Short-range vs mid-range}

In the previous section we used $\mu=0.6$ a.u. $^{-1}$ for our range-separation parameter, similar to $\mu=0.3 \AA^{-1}$ used in HSE and other functionals. But, there is no reason to assume that this value will be the "right" one for MRF. 

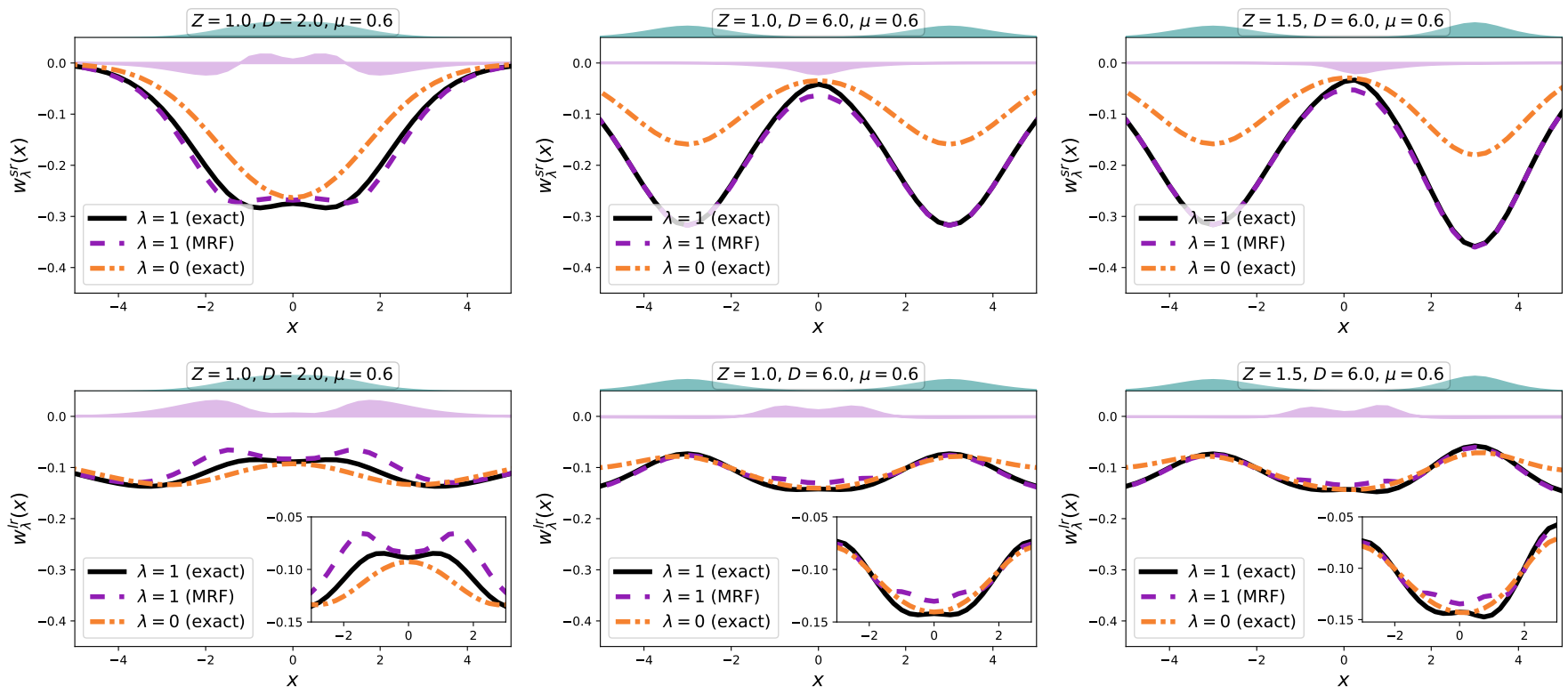

FIG. 7. Plots showing exact range-separated $w_{\lambda}(x)$ - short-range part (top) and long-range part (bottom) for $\mu=0.6-$ energy densities at $\lambda=1$ (black solid lines) and $\lambda=0$ (orange dash-dot) and that of MRF at $\lambda=1$ (purple dashes). Shown are $Z=1, D=2$ (left), $Z=1, D=6$ (left) and $Z=1.5, D=6$ (right). Light purple shaded region indicates deviation of the MRF energy density from the exact one at $\lambda=1$. Densities are shown above the figure (light teal) for illustrative purposes. The inset of the long-range plots zoom into the region of greatest error.
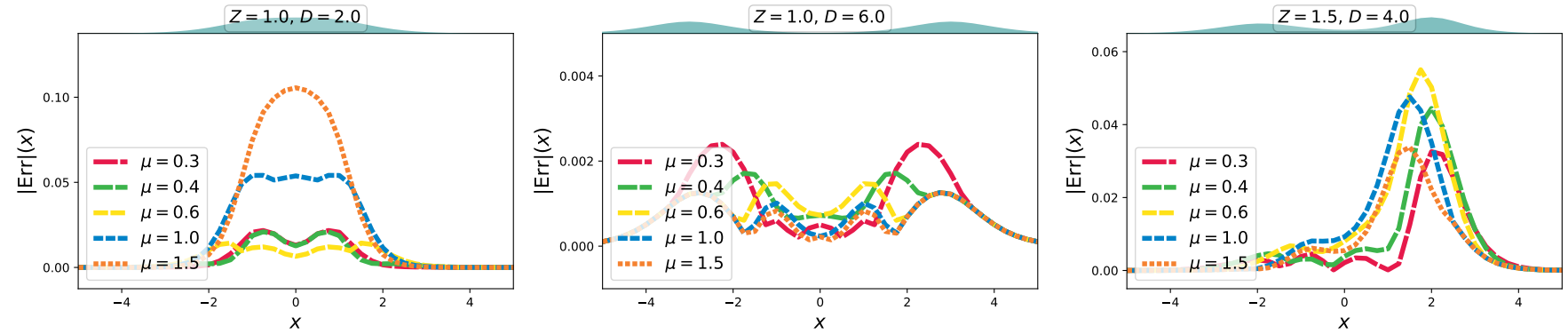

FIG. 8. Errors $|\operatorname{Err}|=n(x)\left[\left|w_{\mathrm{MRF}}^{\mathrm{sr}}-w_{1}^{\mathrm{sr}}\right|+\left|w_{\mathrm{MRF}}^{\mathrm{lr}}-w_{1}^{\mathrm{lr}}\right|\right]$ for various $\mu$, for $Z=1, D=2$ and $Z=1, D=6$ and $Z=1.5, D=4$. We show $D=4$ a.u. rather than 6 a.u. in the last case, to illustrate the most difficult system we found.

Thus, in this section we explore the variation of the energy with $\mu$.

In Figure 8, we show the mean absolute error of the lr and sr energy contribution:

$$
|\operatorname{Err}|=n(x)\left[\left|w_{\mathrm{MRF}}^{\mathrm{sr}}(x)-w_{1}^{\mathrm{sr}}(x)\right|+\left|w_{\mathrm{MRF}}^{\mathrm{lr}}(x)-w_{1}^{\mathrm{lr}}(x)\right|\right],
$$

as a proxy energy measure to help determine the optimal choice of range separation parameter. In $3 \mathrm{D}$, a similar analysis might be better performed by using, e.g., a good GGA for the long-range part. But for 1D systems we do not have access to range-separated GGAs. We show the cases $Z=1$ and $D=2$ and 6 reported previously. But we replace $Z=1.5$ and $D=6$ by the case $Z=1.5$ and $D=4$, for which the MRF pair-density was poorest (right-panel, Figure 3), and which consequently is the most difficult test of MRF that we found.
It is clear from the plots that setting $\mu=0.3$ seems to give the best overall results. It performs similarly to $\mu=0.6$ in the two $Z=1$ cases, but better in the hard $Z=1.5 / D=4$ case. We note that $\mu=0.3$ corresponds to a length scale of 3.3 a.u $(\sim 7 \AA)$, twice the length scale of $\mu=0.6$. For this reason it should probably be classed as mid-range, rather than short-range.

To further explore this dependence, in Figure 9 we show the short-range energy contribution $w^{\mathrm{sr}}$ of the most difficult case $(Z=1.5, D=4)$, with $\mu \in\{0.3,0.6,1.0\}$. It is clear from the plot that the reason that $\mu=0.3$ outperforms its shorter-ranged counterparts is that the error varies in sign, rather than being consistently overand under-estimating. This is not entirely surprising, since the MRF functional is designed around the physics of dissociation, so might be expected to perform best on a length scale relevant to dissociation processes. 

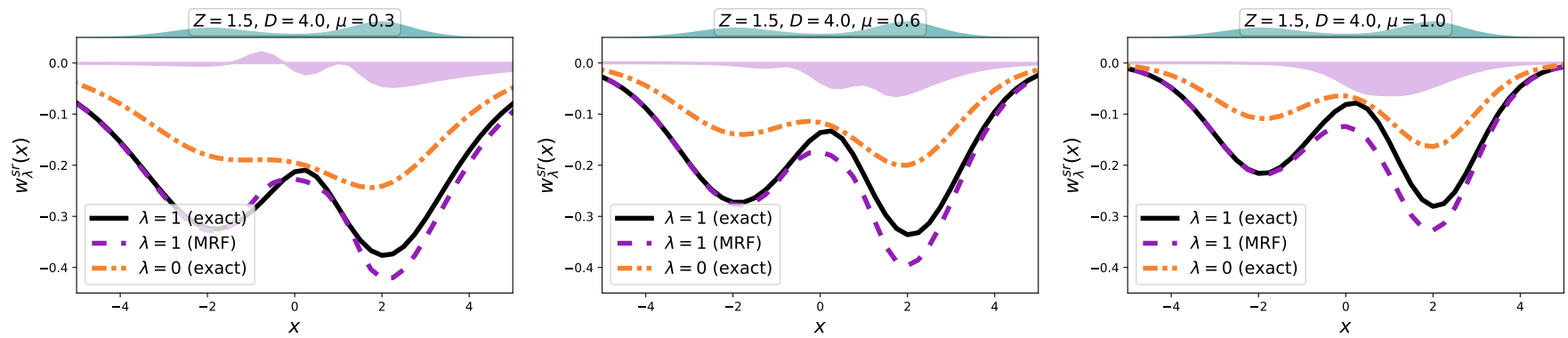

FIG. 9. Like top row of Figure 7 except we set $Z=1.5$ and $D=4$ in all plots, and vary $\mu \in\{0.3,0.6,1.0\}$.

However, before unequivocally supporting a smaller $\mu$ we should consider on important point: 1D molecules provide a useful, relevant and controllable demonstration of dissociation physics, but they do not have the richness of chemistry found in three-dimensions. Thus, while these results do suggest that small values of $\mu$ should not be discounted in future studies on real molecules, they are certainly not conclusive in that regard.

\section{CONCLUSIONS}

In this manuscript we have studied correlations in 1D molecules, using exact results and the multiple radii functional (MRF) and Kohn-Sham (Hartree-exchange, $\lambda=0$ ) approximations. We began by investigating the pairdensity, which showed that MRF could deal with some quite difficult cases. We then focused attention on rangeseparation of energies, in terms of its effect on the energy density and the electronic pair-density.

We found that MRF was, surprisingly, better for shortrange interactions than for long-range ones, at least at the energy level. While this result may seem counterintuitive, in fact it is clear from an illustrative example, and the calculations we performed, that the main contribution to energies is from the correlation hole, which becomes more localised in strongly correlated systems. In this context, the result is less surprising.

What is difficult to pin down from our two-electron one-dimensional molecules is whether or not these effects are predominately short-ranged, or more mid-ranged. If the latter, a double range-separation might be advisable for optimal use of MRF, to divide the energy into shortrange terms to be treated using Hartree-Fock theory, mid-range terms to be treated by MRF, and long-range terms to be treated using semi-local density functional approximations.

In addition to potentially making calculations more accurate, our findings may have practical advantages for implementation of MRF. Unlike the weakly decaying (soft) Coulomb potential, short- and mid-range Coulomb potentials decay quickly. Thus, only radii [from eq. (10)] less than a cutoff radius need to be evaluated. This could potentially lead to more efficient evaluation of MRF en- ergies.

We thus argue that further exploration of rangeseparation in the MRF context is warranted. Especially, range-separation should be investigated in the context of true three-dimensional systems, to better understand the precise length scales that the MRF gets right and wrong. Work along these lines is underway.

\section{REFERENCES}

${ }^{1}$ W. Kohn and L. J. Sham, Phys. Rev. 140, A 1133 (1965).

${ }^{2}$ A. J. Cohen, P. Mori-Sánchez, and W. Yang, Chem. Rev. 112, 289 (2012).

${ }^{3}$ K. Burke, J. Chem. Phys. 136, 150901 (2012).

${ }^{4}$ A. D. Becke, J. Chem. Phys. 140, 18A301 (2014).

${ }^{5}$ A. Pribram-Jones, D. A. Gross, and K. Burke, Annual review of physical chemistry 66, 283 (2015).

${ }^{6}$ N. Q. Su and X. Xu, Annu. Rev. Phys. Chem. 68, 155 (2017).

${ }^{7}$ N. Mardirossian and M. Head-Gordon, Mol. Phys. 115, 2315 (2017).

${ }^{8}$ A. J. Cohen, P. Mori-Sanchez, and W. Yang, Science 321, 792 (2008).

${ }^{9}$ F. Malet and P. Gori-Giorgi, Phys. Rev. Lett. 109, 246402 (2012).

10 A. D. Becke, J. Chem. Phys. 138, 074109 (2013).

${ }^{11}$ S. Vuckovic, T. J. Irons, A. Savin, A. M. Teale, and P. GoriGiorgi, J. Chem. Theory Comput. 12, 2598 (2016).

${ }^{12}$ S. Vuckovic and P. Gori-Giorgi, J. Phys. Chem. Lett. 8, 2799 (2017).

${ }^{13}$ S. Vuckovic, Journal of chemical theory and computation (2019).

${ }^{14}$ M. Seidl, J. P. Perdew, and S. Kurth, Phys. Rev. Lett. 84, 5070 (2000).

${ }^{15}$ M. Seidl, P. Gori-Giorgi, and A. Savin, Phys. Rev. A 75, 042511 (2007).

${ }^{16}$ P. Gori-Giorgi, G. Vignale, and M. Seidl, J. Chem. Theory Comput. 5, 743 (2009).

${ }^{17}$ P. Gori-Giorgi, M. Seidl, and G. Vignale, Phys. Rev. Lett. 103, 166402 (2009).

${ }^{18} \mathrm{~S}$. Vuckovic, L. Wagner, A. Mirtschink, and P. Gori-Giorgi, J. Chem. Theory Comput. 11, 3153 (2015).

${ }^{19}$ K. Burke, F. G. Cruz, and K.-C. Lam, J. Chem. Phys. 109, 8161 (1998).

${ }^{20}$ J. Tao, V. N. Staroverov, G. E. Scuseria, and J. P. Perdew, Phys. Rev. A 77, 012509 (2008).

${ }^{21}$ S. Vuckovic, T. J. P. Irons, L. O. Wagner, A. M. Teale, and P. Gori-Giorgi, Phys. Chem. Chem. Phys. 19, 6169 (2017).

${ }^{22}$ J. Jaramillo, G. E. Scuseria, and M. Ernzerhof, J. Chem. Phys. 118, 1068 (2003).

${ }^{23}$ A. V. Arbuznikov and M. Kaupp, Chem. Phys. Lett. 440, 160 (2007). 
${ }^{24}$ E. Rebolini, R. Izsák, S. S. Reine, T. Helgaker, and T. B. Pedersen, Journal of chemical theory and computation 12, 3514 (2016).

${ }^{25}$ L. O. Wagner, E. M. Stoudenmire, K. Burke, and S. R. White, Phys. Chem. Chem. Phys. 14, 8581 (2012).

${ }^{26}$ F. Malet, A. Mirtschink, K. J. Giesbertz, L. O. Wagner, and P. Gori-Giorgi, Phys. Chem. Chem. Phys. 16, 14551 (2014).

${ }^{27}$ T. Gould, L. Kronik, and S. Pittalis, The Journal of chemical physics 148, 174101 (2018).

${ }^{28}$ D. G. Tempel, T. J. Martínez, and N. T. Maitra, J. Chem. Theory Comput. 5, 770 (2009).

${ }^{29}$ L. O. Wagner, E. Stoudenmire, K. Burke, and S. R. White, Physical review letters 111, 093003 (2013).

${ }^{30}$ S. Giarrusso, S. Vuckovic, and P. Gori-Giorgi, J. Chem. Theory Comput. 9, 3137 (2018).

${ }^{31}$ A. Savin, in Recent Developments of Modern Density Functional Theory, edited by J. M. Seminario (Elsevier, Amsterdam, 1996) pp. 327-357.

${ }^{32}$ J. Toulouse, F. Colonna, and A. Savin, Phys. Rev. A 70, 062505 (2004).

33 J. Heyd, G. E. Scuseria, and M. Ernzerhof, J. Chem. Phys. 118, 8207 (2003).

${ }^{34}$ T. Yanai, D. P.Tew, and N. C.Handy, Chem. Phys. Lett. 393, 51 (2004).

${ }^{35}$ D. C. Langreth and J. P. Perdew, Solid State Commun. 17, 1425
(1975).

${ }^{36}$ O. Gunnarsson and B. I. Lundqvist, Phys. Rev. B 13, 4274 (1976).

${ }^{37}$ M. Levy, Proc. Natl. Acad. Sci. U.S.A. 76, 6062 (1979).

${ }^{38}$ K. Burke, F. G. Cruz, and K.-C. Lam, J. Chem. Phys. 109, 8161 (1998).

${ }^{39}$ S. Vuckovic, M. Levy, and P. Gori-Giorgi, J. Chem. Phys 147, 214107 (2017).

${ }^{40}$ J. P. Perdew and K. Schmidt, in AIP Conference Proceedings, Vol. 577 (AIP, 2001) pp. 1-20.

${ }^{41}$ M. G. Medvedev, I. S. Bushmarinov, J. Sun, J. P. Perdew, and K. A. Lyssenko, Science 355, 49 (2017).

${ }^{42} \mathrm{M}$. Hellgren and T. Gould, "Strong correlation and charge localization in kohn-sham theories with fractional orbital occupations: The role of the potential," (2019).

${ }^{43}$ E. J. Baerends and O. V. Gritsenko, J.Phys. Chem. A 101, 5383 (1997).

${ }^{44}$ W. Koch and M. C. Holthausen, A Chemist's Guide To Density Functional Theory (Wiley-VCH, New York, 2001).

${ }^{45}$ T. M. Henderson, B. G. Janesko, and G. E. Scuseria, The Journal of Physical Chemistry A 112, 12530 (2008), pMID: 19006280, https://doi.org/10.1021/jp806573k. 